\title{
Inherent-opening-controlled pattern formation in carbon nanotube arrays
}

\author{
Xiao Huang ${ }^{1}$, Jijie J Zhou ${ }^{1}$, Elijah Sansom ${ }^{2}$, Morteza Gharib ${ }^{2}$ and \\ Sow Chorng Haur ${ }^{3}$ \\ ${ }^{1}$ Materials Technology, Nanyang Technological University, Nanyang Avenue, 639798, \\ Singapore \\ ${ }^{2}$ Bioengineering, California Institute of Technology, Pasadena, CA 91125, USA \\ ${ }^{3}$ Department of Physics, National University of Singapore, Science Drive, 117542, Singapore \\ E-mail: jjzhou@ntu.edu.sg
}

Received 15 March 2007, in final form 29 May 2007

Published 29 June 2007

Online at stacks.iop.org/Nano/18/305301

\begin{abstract}
We have introduced inherent openings into densely packed carbon nanotube arrays to study self-organized pattern formation when the arrays undergo a wetting-dewetting treatment from nanotube tips. These inherent openings, made of circular or elongated hollows in nanotube mats, serve as dewetting centres, from where liquid recedes from. As the dewetting centres initiate dry zones and the dry zones expand, surrounding nanotubes are pulled away from the dewetting centres by liquid surface tension. Among short nanotubes, the self-organized patterns are consistent with the shape of the inherent openings, i.e. slender openings lead to elongated trench-like structures, and circular holes result in relatively round nest-like arrangements. Nanotubes in a relatively high mat are more connected, like in an elastic body, than those in a short mat. Small cracks often initialize themselves in a relatively high mat, along two or more adjacent round openings; each of the cracks evolves into a trench as liquid dries up. Self-organized pattern control with inherent openings needs to initiate the dewetting process above the nanotube tips. If there is no liquid on top, inherent openings barely enlarge themselves after the wetting-dewetting treatment.
\end{abstract}

\section{Introduction}

Carbon nanotubes (CNTs) are light, strong, thermally stable and chemically inert with a very broad range of applications. A CNT array can be grown on a common substrate through chemical vapour deposition (CVD) methods with individual nanotubes aligned vertically with respect to the substrate [1] Such a CNT array over a large scale is often referred to as a CNT mat when nanotubes are densely packed. Liquidassisted pattern formation in CNT mats has been studied previously from such aspects as capillary forces, van der Waals interactions [2-4], nanotube aspect ratio, site density, solvent surface tension and rate of evaporation $[5,6,3]$. Control of such a self-organized pattern formation may enable low-cost and large-scale applications of carbon nanotubes [7, 8]. In this work, we introduce void spaces of different configurations into $\mathrm{CNT}$ mats and employ them as dewetting centres in order to control the pattern formation.

\section{Experimental methods}

\subsection{Growth of carbon nanotubes}

Growth of carbon nanotubes is a bottom-up self-assembly process. Configuration of a CNT array is controllable through its substrate and catalyst layer. This work uses three set-ups for the nanotubes. (i) The catalyst used for aligned carbon nanotubes is highly pure nickel metal. The nickel film is formed on a chromium-coated substrate and then nucleates into nanoscale particles at the beginning of nanotube growth. After nanotube growth, the small nickel particles are lifted up to the tips of carbon nanotubes. The average tube diameter is $100 \mathrm{~nm}$ and the height varies from 7 to $30 \mu \mathrm{m}$ with growth time (courtesy of Dr Zhongping Huang, NanoLab Incorporation, MA). (ii) The catalyst is iron, coated on thermal oxide silicon or quartz. After $\mathrm{CNT}$ growth at $650{ }^{\circ} \mathrm{C}$ with acetylene, iron remains at the tube root. The average tube diameter is $20 \mathrm{~nm}$. 

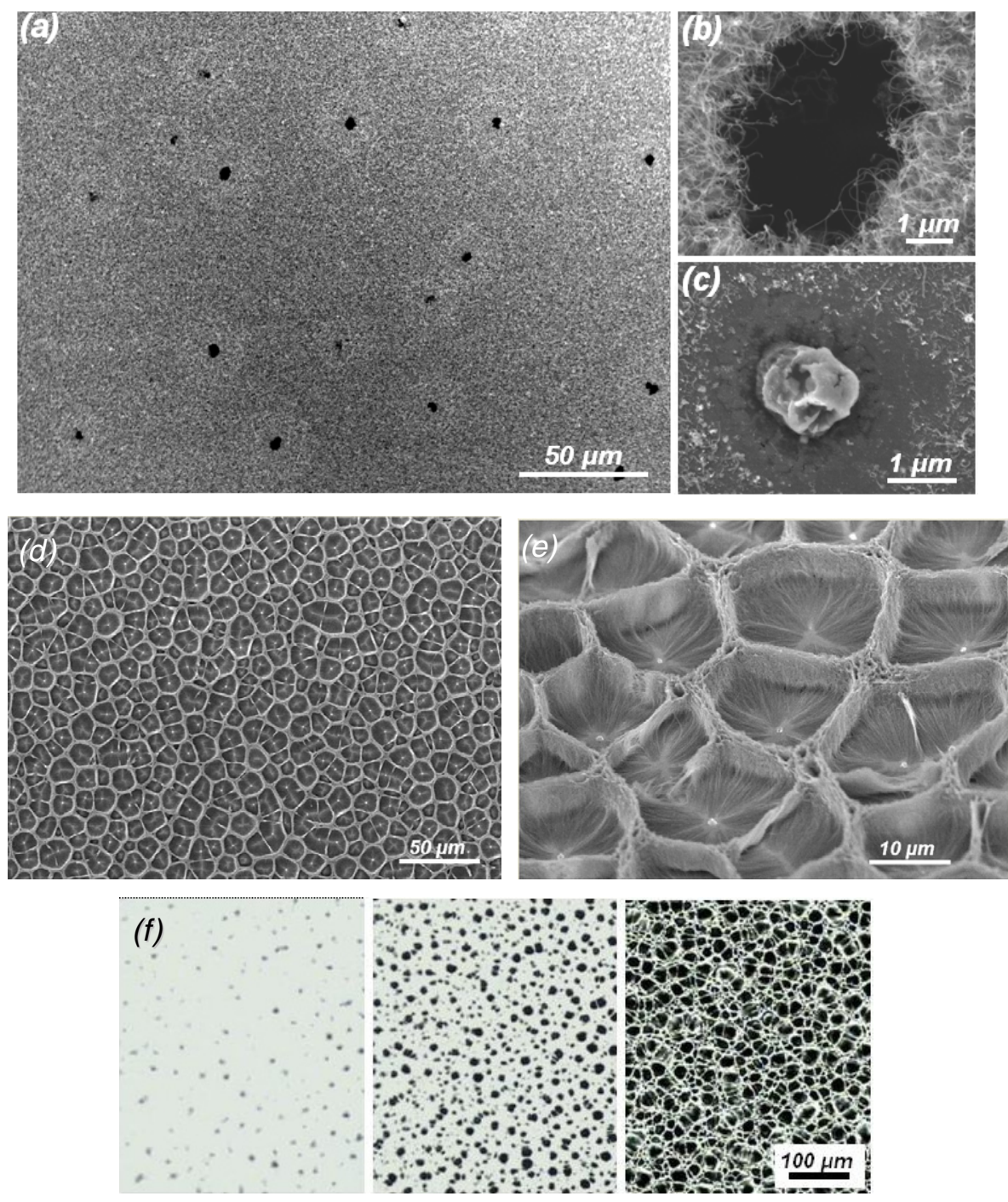

Figure 1. Inherent circular openings in a $12 \mu \mathrm{m}$ high CNT array, and these openings induced pattern formation. (a) Top view of the as-grown array with inherent micro-holes; (b) close-up of a micro-hole; (c) an aluminium particle introduced during sputtering; (d) large-area self-organization of polygonal nest-like structures; (e) aluminium particles: the bright dots, are at the centres of these nests; (f) pattern evolution in a time course. The inherent openings gradually enlarge themselves with the aid of liquid.

The array height varies from 3 to $60 \mu \mathrm{m}$ depending on growth time (courtesy of Dr Michael J Bronikowski, Jet Propulsion Laboratory, CA). (iii) The catalyst is iron on aluminium sputtered Si wafers. The synthesis, taking place at $750{ }^{\circ} \mathrm{C}$ with ethylene as carbon source, results in multi-wall CNT arrays with tube diameters of 30-40 $\mathrm{nm}$ and array heights adjustable in between 9 and $200 \mu \mathrm{m}$ (CVD system is ET2000, First Nano, CA).

\subsection{Inherent openings are made in CNT mats}

Aluminium micro-particles are introduced onto the substrates during sputtering of the $\mathrm{Al}$ underlayer in type (iii) set-up. After CVD growth of CNTs, no nanotubes grow on such particles. Round openings, rooted on these particles, are seen as cylindrical voids in CNT mats, as illustrated in figures 1(a)(c). The diameter of these openings is approximately $3 \mu \mathrm{m}$ or bigger; the distance between adjacent openings varied from 10 to $60 \mu \mathrm{m}$. Elongated openings originated from a wafer defect as in figures 2(a)-(c), thermal fractures in an oxide layer as in figures 2(d)-(h) or laser-pruned (explained in section 2.4) deep grooves as in figures 3 and 4(f). The width of these elongated openings is approximately $3 \mu \mathrm{m}$; the length extends from $10 \mu \mathrm{m}$ all the way to $1 \mathrm{~mm}$.

\subsection{Self-organization among CNTs is induced with a small amount of liquid}

Liquid is introduced through placing a droplet on top of a CNT mat and then leaving it to dry in air. Liquid volume is no more than $10 \mu \mathrm{l}$ in such a wetting-and-dewetting treatment for pattern formation among CNT arrays. The volume is measured with Hamilton syringes. The droplet is suspended from a Teflon tip of a Hamilton syringe before it is slowly moved down and placed atop CNTs. The droplet is assumed stationary with no injecting speed. In order to convey waterbased solutions through hydrophobic carbon nanotubes, we use surfactant aqueous solutions. The droplet spreads outwards 
(a) (b) $\underline{50 \mu m}$
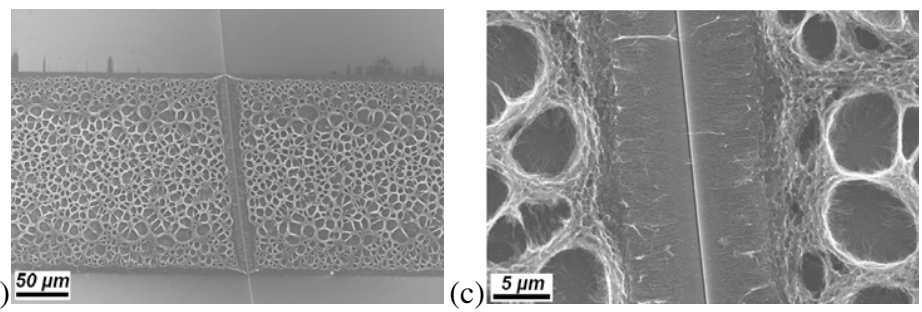

(d)

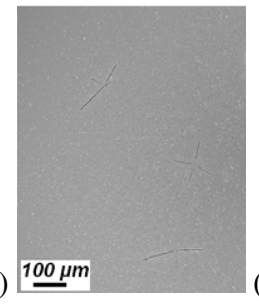
(e) $20 \mu \mathrm{m}$
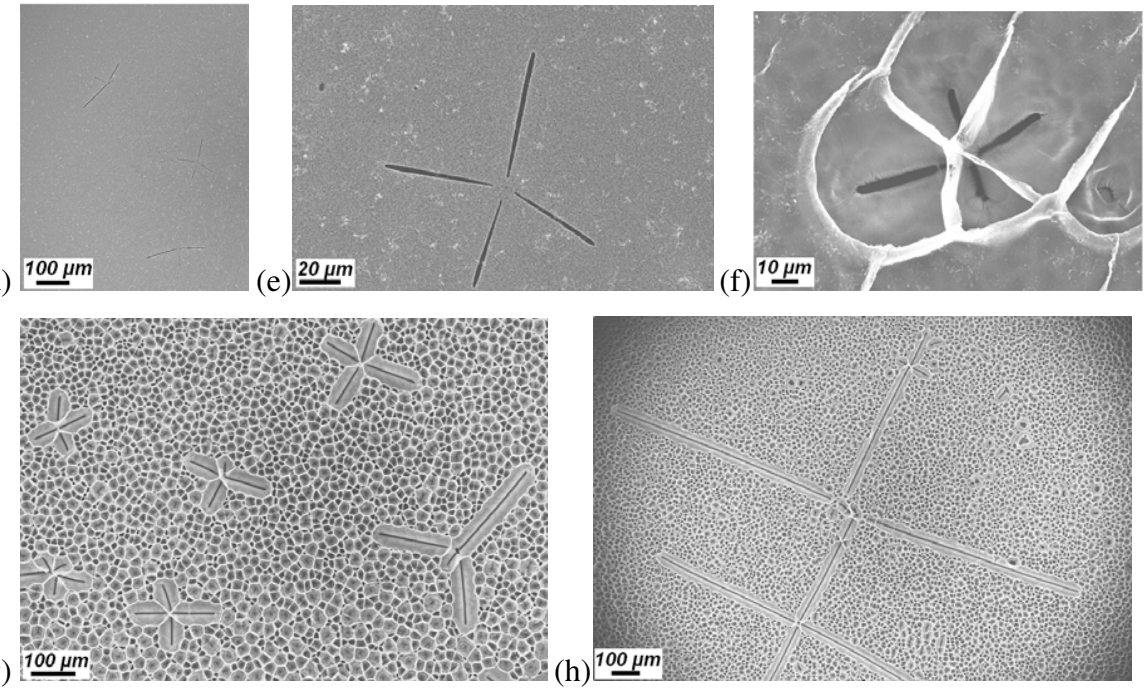

(h) $100 \mu \mathrm{m}$

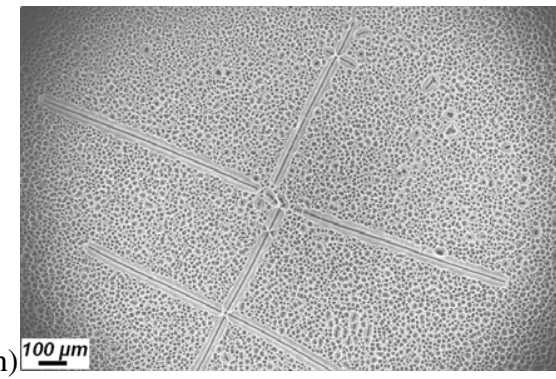

Figure 2. Inherent elongated openings and their post-treatment trenches. The average width of the openings here is around $3 \mu \mathrm{m}$ and length varies from $10 \mu \mathrm{m}$ to $1 \mathrm{~mm}$. (a), (d) and (f): Inherent elongated voids in CNT mats before liquid treatment; others: post-treatment. (a)-(c): A straight line in a $7 \mu \mathrm{m}$ high CNT array; (d)-(h) thermal-fracture-induced slender voids in an as-grown $40 \mu \mathrm{m}$ high CNT mat and their open-up trench-like structure after liquid treatment. In the special case of (f), because the heights of the nanotubes are lager than the lengths of the four inherent voids, the final open-up structure is more round and polygonal, dissimilar to the original slender shape.

and forms a thin liquid film on top of the CNT mat. We only exercise stable spreading [9] with $0.18 \mathrm{wt} \%$ sodium dodecyl sulfate (SDS) for this study. The wetting and dewetting processes are captured under optical microscopes. Initial and final configurations are characterized with field emission scanning electron microscopes (JSM-6340F).

\subsection{Step structures are created to restrict flow}

Step structures are created to restrict flow in a control experiment. Such structures as illustrated in figure 3 are made with a laser-pruning method [10]. For pruning, an as-grown dense CNT mat is placed on a computer-controlled X-Y stage. A laser beam, focusing on the top surface of the CNT mat, burns off CNT tips and removes a thin layer of the CNT mat as the stage moves. The thickness of removal depends on dwell time and laser power [10].

\section{Results and discussion}

Through our liquid treatment method, the self-organized pattern formation in CNT arrays is controlled with inherent openings. Liquid withdraws spontaneously away from these openings, as liquid seeps into the arrays and evaporates. Liquid withdrawal leads to the expansion of dry zones [11]. When the dry zones expand and liquid recedes away from the openings, nanotubes near these openings are pulled away at the liquid/air interface by surface tension and form a self-organized pattern.
In our particular process of encouraging self-organization, a small amount of liquid is placed on top of dense CNT mats. This is different from immersing the whole mat into bulky liquid. A liquid droplet forms a thin liquid film on CNT tips before interstitial air in the CNT mat is fully displaced or absorbed [9]. As the thickness of this liquid film decreases, the film conforms its shape to its underneath surface morphology. The liquid film gradually deforms into a pattern that largely matches the pre-treatment pattern of the dry mat. Inherent openings further facilitate such liquid deformation by draining liquid down quickly through the openings. Comparing with seeping into dense interstices among CNTs, flow through micron openings experiences more volumetric space and less viscous drag. Therefore, liquid preferably seeps into the mat through the inherent openings and initiates dry zones therein.

\subsection{Self-organized pattern formation}

The self-organized process is in a sequence of (i) nucleation of dry zones at the circular openings, (ii) enlargement of the openings accompanied by nanotube deformation, (iii) formation of nest-like structures when nanotubes between adjacent openings are compressed into thin ridges and the openings stop growing. A series of optical micrographs in figure 1(f) show the pattern formation induced by circular openings in a $12 \mu \mathrm{m}$ high array. The resulting nest-like structures for the $12 \mu \mathrm{m}$ array are in the shape of polygons with ridges perpendicular to the substrate as illustrated in 


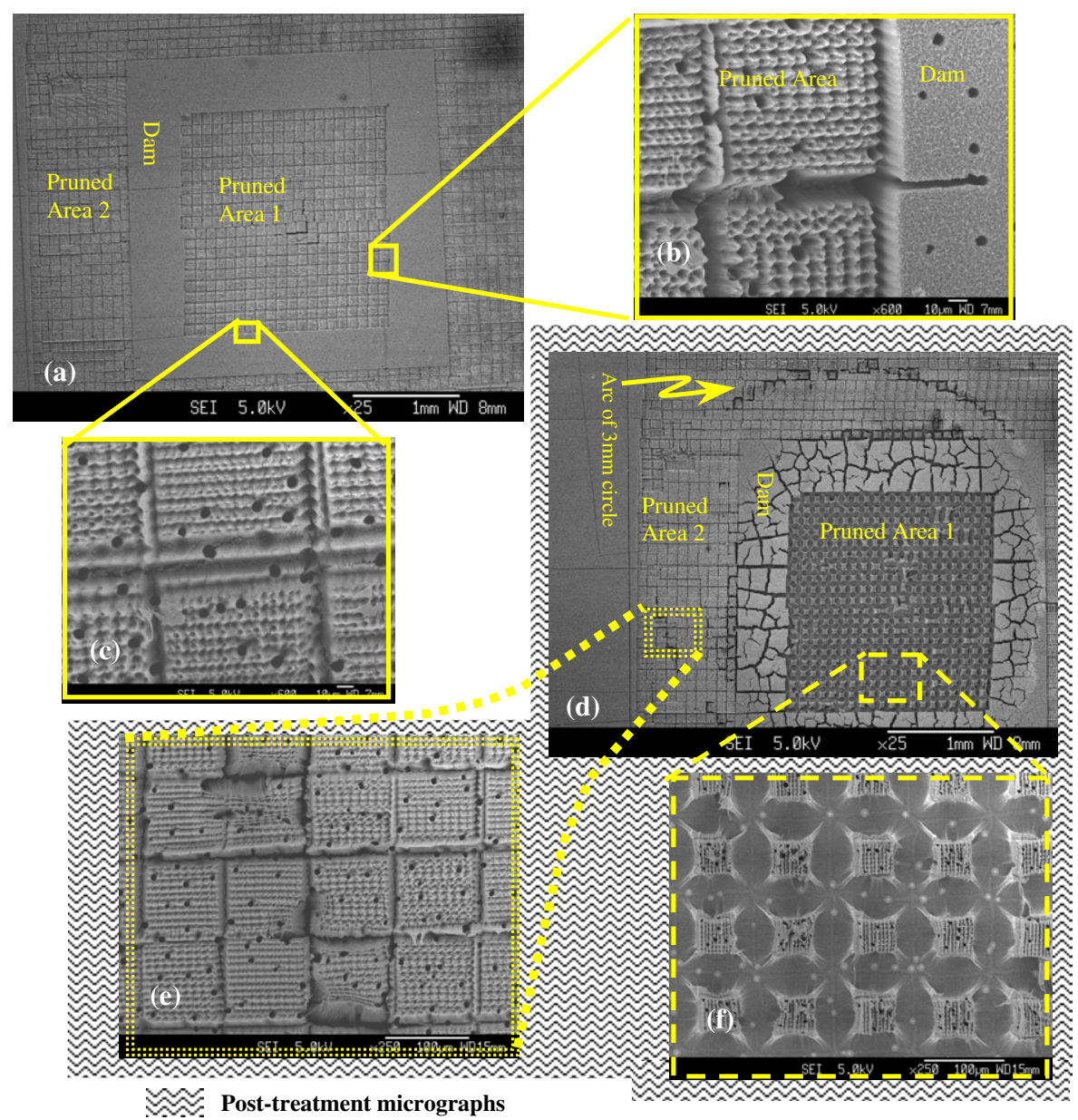

Figure 3. Different pattern formation in the presence and absence of a liquid film on top. The pruned areas are separated by a dam-like structure. Micrograph (b) is a $15^{\circ}$ tilted view, all others are top view. (a)-(c): SEM micrographs before liquid treatment. The laser-pruned areas are made up of $100 \mu \mathrm{m}$ squares, separated by grooves. (d)-(f): Post-treatment micrographs. Cracks are seen along arc segments of a $3 \mathrm{~mm}$ circle around the dam. The top arc is indicated with an arrow in (d). Other than the crack, the pre-treatment and post-treatment morphologies are almost identical in pruned area 2 by comparing (c) and (e), but quite different in pruned area 1 when comparing (c) and (f). (This figure is in colour only in the electronic version)

figure 1(e). The original location of each circular opening is labelled with an aluminium micro-particle at its root, because each inherent opening results from an $\mathrm{Al}$ particle that is induced during preparation of the substrate. After our wetting and dewetting treatment, an $\mathrm{Al}$ particle is mostly seen at the centre of each nest, as demonstrated in figures $1(\mathrm{~d})$ and (e). As a consequence, we infer that these circular openings serve as dewetting centres during the self-organized pattern formation.

In order to further prove that inherent openings in dense CNT arrays can control the pattern formation, we introduce elongated inherent openings in CNT mats. After an aqueous droplet is placed on top, dry zones initialize from these elongated openings and produce self-organized trench-like structures. These post-treatment structures are very consistent with arrangements of the slender openings in pre-treatment mats as exemplified in figure 2. On places without introduced openings, round or polygonal nests are freely assembled in the mats. These freely assembled nests have been studied as drying-induced pattern formation in nanotube arrays in several previous works $[12,8,13,6,14-16]$. Even though our liquid dynamic treatment takes a different approach, our work supports Liu et al's conclusion [3] that the aligned nanotubes bend from lower to higher density regions. They reach this conclusion through investigating the capillary-force-induced hydrostatic dilation stress.

\subsection{Function of liquid films above nanotube tips}

A control experiment is conducted to contrast self-organized patterns with and without a free liquid film on top of nanotube arrays. It is shown in the following paragraphs that inherent openings can only control pattern formation where such a liquid film exists.

We prune CNTs in two areas with lasers and leave CNTs in between these two areas intact. The in-between CNTs are $130 \mu \mathrm{m}$ high. They protrude as a dam, and are named so. Both areas inside and outside the dam structure are made by sequentially cutting $100 \mu \mathrm{m}$ squares as in figures 3(a)-(c). Due to repeated laser exposure, deep grooves are created at the boundaries of the squares. Inside each square, small grooves are seen as an indicator of the pathway that a laser beam has travelled. The laser-pruned CNTs, i.e. both inside and outside 
(a)

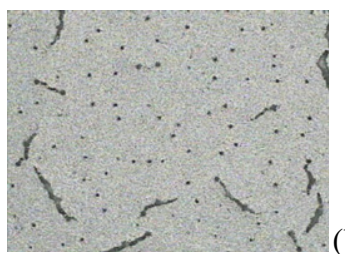

(b)

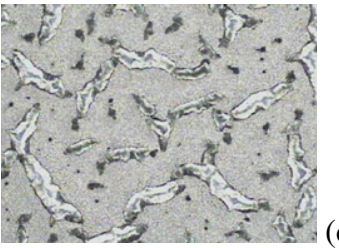

(c)

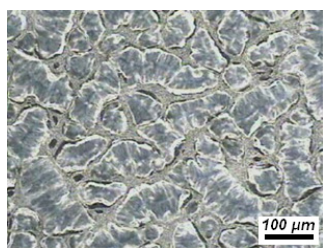

(d)
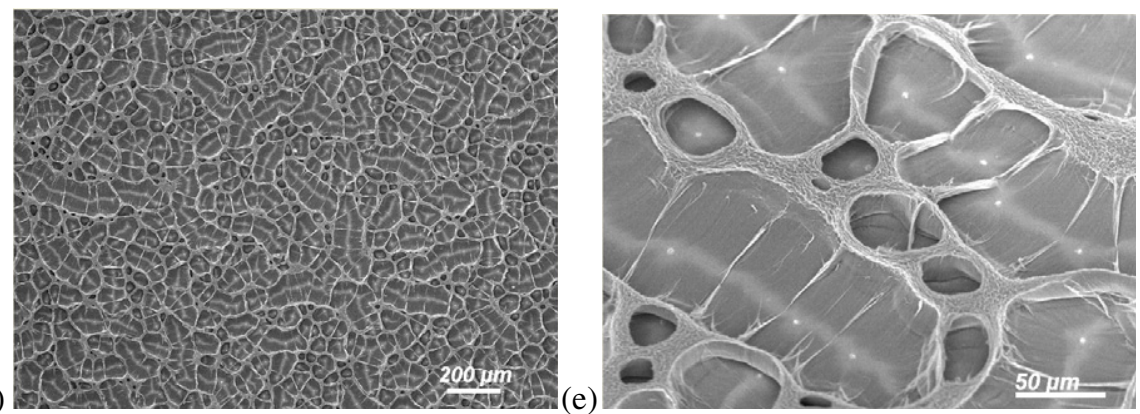

Figure 4. Micropattern formation on 70 and $130 \mu \mathrm{m}$ high CNT arrays. (a)-(e): Self-organization is induced with SDS aqueous solution on a $70 \mu \mathrm{m}$ array. (a)-(c). Optical micrographs of self-organized patterns in progress. (a): Microcracks initialized along adjacent round openings. (b): The microcracks become widened. (c): Trench-like cells are formed with underdeveloped circular nests in between. (d)-(e): SEM images of the finishing pattern.

the dam, are 30-40 $\mu \mathrm{m}$ shorter than the intact ones. Within the pruned areas, the deep grooves separating the $100 \mu \mathrm{m}$ squares are $30-40 \mu \mathrm{m}$ deep, and approximately $20 \mu \mathrm{m}$ wide, that is, the nanotubes in the grooves are approximately $70 \mu \mathrm{m}$ shorter than the intact ones in the dam. After a liquid droplet is placed inside the dam, liquid surface spreading cannot jump over the dam structure, because of the step height of the dam [9]. Only interstitial flow advances out of the dam.

After such a liquid treatment process, different flow conditions lead to different patterns on the pruned areas. Inside the dam, structure is a regular array of condensed squares (pruned area 1 in figure 3(f)). Each condensed square consists of a compact cluster of nanotubes, which results from bending of the nanotubes and enlargement of deep grooves as the nanotubes are pulled away from the grooves by liquid. Opening up from the deep grooves dominates the potential enlargement of other dewetting centres such as inherent round openings and small grooves on laser pathways. Because these deep grooves are relatively wide, deep and continuous over a long distance, when they expand in width, other inferior openings are forced to close up if they ever tried to enlarge.

Outside the dam, the deep grooves are not significantly enlarged by interstitial liquid. Except at a liquid front, the pretreatment (figure 3(c)) and post-treatment (figure 3(e)) surface morphologies out of the dam are not much different, especially when comparing pruned area 2 in figure 3(e) with significantly reorganized pruned area 1 in figure 3(f). The front of interstitial liquid is tracked with cracks along the arc of a $3 \mathrm{~mm}$ circle that is pointed out in figure $3(\mathrm{~d})$. These cracks correspond to the final position of the liquid front inside the CNT mat before liquid recedes. Because liquid only exists on one side of such a liquid front, nanotubes are pulled toward the liquid as it evaporates, and therefore cracks appear. Inside the crack encircled area but outside the dam structure, no inherent circular openings or pruned grooves lead to conformational reorganization of CNTs. Therefore, activation of dewetting centres with a thin liquid film on top of the mat is a key factor for control of pattern formation.

\subsection{Cracks form from adjacent round openings in high CNT mats}

A linear relationship between CNT array height and feature size of a self-organized pattern is reported by Chakrapani et al [6]. They explain this relationship by theoretical studies of crack pattern formation using spring-block models. A critical value between definitions of 'high' for elastic-body behaviours and 'short' for less elastic-body interaction depends on the site density, feature dimension of the inherent opening and other potential geometric parameters of CNTs as well. From our observation with $3 \mu \mathrm{m}$ openings in dense arrays of site density $10^{10}-10^{11} \mathrm{MWNT} \mathrm{\textrm {cm } ^ { - 2 }}$, elastic-body-like crack formation is not observed for mats with a height below $50 \mu \mathrm{m}$ : therefore, 'below $50 \mu \mathrm{m}$ ' is roughly considered as 'short' and 'above $50 \mu \mathrm{m}$ ' is relatively 'high'.

Configuration and location of inherent openings are preserved in short nanotube arrays during self-organization as shown in figures 1(e), 2(c), (g) and (h), and 3(e). In relatively high CNT arrays, or with relatively small inherent openings, the post-treatment self-organized structures are probably dissimilar to the pre-treatment shape of inherent openings, e.g. figure 2(f), even if self-organization is initially induced by the inherent openings. On relatively high CNT mats with round inherent openings, dry zones are initialized randomly along adjacent openings to first form microcracks as exemplified in figure 4(a). The cracks propagate longitudinally before running into another, and widen themselves laterally as in figures 4(b) and (c). Those openings, not involved in microcrack formation, are enlarged separately to a variety of extents with a maximum diameter of $100 \mu \mathrm{m}$ in a $70 \mu \mathrm{m}$ mat as shown in figure 4(e). When the microcracks grow into a width of about $100 \mu \mathrm{m}$, enlargement of these separate round openings becomes tangible under an optical microscope. 


\section{Summary}

Self-organized pattern formation of carbon nanotube arrays is promoted with a wetting-dewetting treatment method. The pattern formation is controlled with inherent openings in the arrays. These inherent openings serve as dewetting centres when liquid recedes away from them. Nanotubes are pulled away from the dewetting centres by liquid surface tension during self-organization. Among short nanotubes, the selforganized patterns are consistent with the shape of the inherent openings. In a relatively high mat, small cracks often initialize themselves along two or more adjacent round openings. A liquid film on top plays a significant role in controlling pattern formation with inherent openings. Without liquid on top, inherent openings barely enlarge themselves by interstitial liquid.

\section{Acknowledgment}

This work is supported by Academic Research Fund (grant no. RG27/05) from Ministry of Education, Singapore.

\section{References}

[1] Fan S S, Chapline M G, Franklin N R, Tombler T W, Cassell A M and Dai H J 1999 Science 283 512-4
[2] Nguyen C V, Delzeit L, Cassell A M, Li J, Han J and Meyyappan M 2002 Nano Lett. 2 1079-81

[3] Liu H, Li S, Zhai J, Li H, Zheng Q, Jiang L and Zhu D 2004 Angew. Chem. Int. Edn 43 1146-9

[4] Lau K K S, Bico J, Teo K B K, Chhowalla M, Amaratunga G A J, Milne W I, McKinley G H and Gleason K K 2003 Nano Lett. 3 1701-5

[5] Zhao Y P and Fan J G 2006 Appl. Phys. Lett. 88 103123-3

[6] Chakrapani N, Wei B, Carrillo A, Ajayan P M and Kane R S 2004 Proc. Natl Acad. Sci. 101 4009-12

[7] Futaba D N et al 2006 Nat. Mater. 5 987-94

[8] Correa-Duarte M A, Wagner N, Rojas-Chapana J, Morsczeck C, Thie M and Giersig M 2004 Nano Lett. $42233-6$

[9] Zhou J J, Noca F and Gharib M 2006 Nanotechnology 17 4845-53

[10] Lim K Y, Sow C H, Lin J Y, Cheong F C, Shen Z X, Thong J T L, Chin K C and Wee A T S 2003 Adv. Mater. 15 300-3

[11] Gennes P-G d, Brochard-Wyart F and Quere D 2004 Capillarity and Wetting Phenomena: Drops, Bubbles, Pearls, Waves (New York: Springer Science+Business Media)

[12] Li Q W, DePaula R, Zhang X F, Zheng L X, Arendt P N, Mueller F M, Zhu Y T and Tu Y 2006 Nanotechnology 17 4533-6

[13] Sheng L M, Liu M, Liu P, Wei Y, Liu L and Fan S S 2005 Appl. Surf. Sci. 250 9-13

[14] Zhao Z B, Qu J Y, Qiu J S, Wang X Z and Wang Z Y 2006 Chem. Commun. 594-6

[15] Liu H, Zhai J and Jiang L 2006 Soft Matter 2 811-21

[16] Sidorenko A, Krupenkin T, Taylor A, Fratzl P and Aizenberg J 2007 Science 315 487-90 